\title{
Some topological properties of certain Riemannian manifolds with positive curvature
}

\author{
By Toshio Sakaguchi
}

\section{Introduction.}

Let $M$ be a compact and simply connected Riemannian manifold with positive curvature $K, 0<K \leqq 1$. Denote by $d(p, q)$ the distance between two points $p$ and $q$ of $M$. K. Hatsuse has introduced the following number $L(M)$;

$$
L(M)=\operatorname{Max}_{p, q, r \in M}\{d(p, q)+d(q, r)+d(r, p)\} .
$$

Actually it is possible to define $L(M)$ for any compact and connected Riemannian manifold with positive curvature. It has been studied by $\mathrm{K}$. Hatsuse and Y. Tsukamoto [4],* [5] to investigate the topological structure of $M$ satisfying suitable conditions for $L(M)$. In particular, K. Hatsuse has proved the following theorem.

TheOREM. Let $M$ be a compact and simply connected Riemannian manifold with positive curvature $K, 0<K \leqq 1$. If $L(M)<3 \pi$, then $M$ is homeomorphic to a sphere. In particular, if $L(M)=2 \pi$, then $M$ is isometric to the sphere with constant curvature 1.

The purpose of the present paper is to prove the following theorems.

Theorem A. Let $M$ be a compact and connected Riemannian manifold with positive curvature $K, 0<K \leqq 1$. If $L(M)=2 \pi$ and there exist two points $p$ and $q$ of $M$ satisfying $d(p, q)=\pi$, then $M$ is isometric to the sphere with constant curvature 1.

THEOREM B. Let $M$ be an $n$-dimensional ( $n \geqq 2)$ compact and connected Riemannian manifold which is not simply connected. Suppose that the sectional curvature $K$ of $M$ satisfies the inequalities $1 / 4<\delta \leqq K \leqq 1$, where $\delta$ is a constant, and the fundamental group $\pi_{1}(M)$ of $M$ satisfies $\pi_{1}(M)=Z_{2}$. If $L(M)=3 \pi / 2$, then $M$ is isometric to the real projective space $P R^{n}(1)$ of constant curvature 1 , and if $L(M)=3 \pi / 2 \sqrt{\delta}$, then $M$ is isometric to $P R^{n}(\delta)$ of constant curvature $\delta$.

$\S 1$ will be of reviews of definitions and notations and $\S 2$ will be devoted

* Numbers in brackets refer to the references at the end of the paper. 
to some known results to use later. And in $\S 3$, the theorems A and B shall be proved.

The present author wishes to express his deep appreciation to Professor Yoshie Katsurada and Doctor Tamao Nagai for their constant guidances and criticisms.

\section{§1. Definitions and notations.}

Let $M$ be an $n$-dimensional $(n \geqq 2)$ complete and connected Riemannian manifold of class $C^{\infty}$ without any other statement and $M_{p}$ the tangent space at the point $p$ of $M$. We denote by \|\|$($ resp. $<,>$ ) the norm (resp. scalar product) which defines the Riemannian structure of $M$. We denote by $\rho=$ $\rho(X, Y)$ the plane section spanned by two linearly independent vectors $X$ and $Y$ belonging to the tangent space $M_{p}$, and by $K(\rho)=K(X, Y)$ the sectional curvature corresponding to a plane section $\rho=\rho(X, Y)$, which is by definition, the scalar

$$
K(\boldsymbol{\rho})=K(X, Y)=-\frac{<R(X, Y) X, Y>}{\|X\|^{2}\|Y\|^{2}-<X, Y>^{2}}
$$

where $R$ denotes the Riemannian curvature tensor on $M$. In a compact manifold with positive curvature, it is always possible that we have the norm of Riemannian structure of $M$ such that $0<K(\rho) \leqq 1$ for any plane section $\rho$. We denote by $S^{n}(1)$ an $n$-dimensional sphere with constant curvature 1 . We consider that all the geodesics on $M$ and $S^{n}(1)$ are parametrized by the arc length measured from their origin. If $\Lambda=\{\lambda(s)\}\left(0 \leqq s \leqq s_{0}\right)$ is such a geodesic, then $\lambda^{\prime}(s)$ denotes its tangent vector at $\lambda(s)$ and we have $\left\|\lambda^{\prime}(s)\right\|=1$ for all $s$. We denote by $d(p, q)($ resp. $\hat{d}(\hat{p}, \hat{q}))$ the distance between two points $p$ and $q$ of $M$ (resp. $\hat{p}$ and $\hat{q}$ of $S^{n}(1)$ ), with respect to the metric structure associated canonically to its Riemannian structure (resp. canonical metric of $\left.S^{n}(1)\right)$. For convenience' sake we put " $\wedge$ " over the elements of $S^{n}(1)$ corresponding to the elements of $M$ without any other statement. If the manifold $M$ is compact, we denote by $d(M)$ its diameter, that is the least upper bound of $d(p, q)$ when $p$ and $q$ vary on $M$. The geodesic joining $p$ to $q$ is called the shortest geodesic segment if its length is equal to $d(p, q)$. By the cut locus $C(p)$ of a point $p$ of $M$, we mean the set of points $\{q\}$, on geodesics starting at $p$, where each point $q$ is the last point for which the geodesic segment $p q$ from $p$ to $q$ realizes the absolute minimum of the length for all segments of curves joining $p$ to $q$. By the geodesic triangle here we always mean a geodesic triangle composed of three shortest geodesic segments. We denote by $\triangle(p, q, r)$ the geodesic triangle 
whose vertices are $p, q$ and $r$, and $\Varangle p=\Varangle(q, p, r)$ the angle between the geodesic segments $p q$ and $p r$.

\section{§2. Reviews of the known results.}

We shall devote the following results which are necessary to prove our theorems.

Theorem 1. (H. Nakagawa [1]) If, in an n-dimensional ( $n \geqq 2)$ complete and connected Riemannian manifold $M$, there exists a point $p$ such that $C(p)$ consists of only one point $q$ and $d(p, q)=\pi$, then $M$ is isometric to an n-dimensional sphere $S^{n}(1)$ with constant curvature 1 .

THEOREM 2. (Y. Tsukamoto [3]) Let $M$ be a Riemannian manifold with positive curvature $K, 0<K \leqq 1$. Denote by $\triangle(\hat{p}, \hat{q}, \hat{r})$ the geodesic triangle on the sphere $S^{n}(1)$ with constant curvature 1 whose side lengths are equal to the corresponding side lengths of the geodesic triangle $\triangle(p, q, r)$ on $M$. If $\triangle(p, q, r)$ is contained in a convex neighbourhood, then each angle of $\triangle(p, q, r)$ is not greater than the corresponding angle of $\triangle(\hat{p}, \hat{q}, \hat{r})$.

THEOREM 3. (K. Shiohama [2]) Let $M$ be an n-dimensional $(n \geqq 2)$ complete and connected Riemannian manifold which is not simply connected. Suppose that the sectional curvature $K$ of $M$ satisfies the inequalities $1 / 4<$ $\delta \leqq K \leqq 1$, where $\delta$ is a constant, and the fundamental group $\pi_{1}(M)$ of $M$ satisfies $\pi_{1}(M)=Z_{2}$. Then for any point $p$ of $M, \pi / 2 \leqq d(p, C(p)) \leqq \pi / 2 \sqrt{\delta}$ and $\pi / 2 \leqq d(M) \leqq \pi / 2 \sqrt{\delta}$, where the left hand side equalities hold if and only if $M$ is isometric to the real projective space $P R^{n}(1)$ of constant curvature 1 , and the right hand side equalities hold if and only if $M$ is isometric to $P R^{n}(\hat{\boldsymbol{o}})$ of constant curvature $\boldsymbol{\delta}$.

\section{§3. Proof of the theorems.}

In order to prove theorem $A$, we prepare the following two lemmas.

LemMa 1. Let $M$ satisfy the assumptions of theorem $A$. Then for any two points $r$ and $s$ of $M$, we have the inequality $d(r, s) \leqq \pi$.

PRoof. By the definition of $L(M)$ we have

$$
d(r, s)+d(s, t)+d(t, r) \leqq L(M)
$$

for any three points $r, s$ and $t$ of $M$. Therefore, from $L(M)=2 \pi$, we have

$$
d(r, s) \leqq d(r, t)+d(t, s) \leqq 2 \pi-d(r, s) .
$$

Hence we have $d(r, s) \leqq \pi$.

Q.E. D.

Lemma 2. Let $M$ satisfy the assumptions of theorem $A$. Then for 
any three points $r, s$ and $t$ of $M$, we have one of the following two cases.

(i) One of the three numbers $d(r, s), d(r, t)$ and $d(t, s)$ is equal to $\pi$. And if $d(r, s)=\pi$, we have the equality $d(r, t)+d(t, s)=\pi$.

(ii) All the three numbers $d(r, s), d(r, t)$ and $d(t, s)$ are less than $\pi$.

Rroof. From $L(M)=2 \pi$ and lemma 1 , we can easily see that either the first part of the case (i) or the case (ii) can only occur. Therefore we must prove the second part of the case (i). By the same argument as the proof of lemma 1, we have the inequalities

$$
d(r, s) \leqq d(r, t)+d(t, s) \leqq 2 \pi-d(r, s) .
$$

From $d(r, s)=\pi$ we have the equality

$$
d(r, t)+d(t, s)=\pi \text { Q. E. D. }
$$

Proof of Theorem A. The proof would be complete by virtue of theorem 1 if we could show that $C(p)$ consists of only one point $q$. By lemma 1 we have $d(M) \leqq \pi$. Therefore, by the definition of the cut locus and $d(p, q)=\pi$, we have that $q$ belongs to $C(p)$. We assume now that $C(p)$ does not coincide with $\{q\}$. Then, taking account of the properties of the cut locus, we see that there is a convex neighbourhood $U$ of $q$ such that the intersection of $U-\{q\}$ and $C(p)-\{q\}$ is not empty. For any point $r$ in $U_{\cap} C(p)-\{q\}$, let $s$ be the point on the shortest geodesic segment joining $p$ to $r$ which is sufficiently close to $r$ and contained in $U$. Thus we obtain the geodesic triangle $\triangle(q, r, s)$ which is contained in $U$. Let $\triangle(\hat{q}, \hat{r}, \hat{s})$ be a geodesic triangle on the sphere $S^{n}(1)$ of constant curvature 1 which has the same side lengths as $\triangle(q, r, s)$. Then, by theorem 2 we have $\Varangle \hat{q} \geqq \Varangle q$, $\Varangle \hat{r} \geqq \Varangle r$ and $\Varangle \hat{s} \geqq \Varangle s$. On the other hand, by $d(p, q)=\pi$ and lemma 2 , we get

$$
d(p, s)+d(s, r)+d(r, q)=d(p, r)+d(r, q)=\pi=d(p, s)+d(s, q) .
$$

So we have

$$
d(s, r)+d(r, q)=d(s, q)=\pi-d(p, s)<\pi .
$$

Therefore we have

$$
\hat{d}(\hat{s}, \hat{r})+\hat{d}(\hat{r}, \hat{q})=\hat{d}(\hat{s}, \hat{q})<\pi .
$$

Hence the equalities $\Varangle \hat{q}=0, \Varangle \hat{r}=\pi$ and $\Varangle \hat{s}=0$ hold and imply $\Varangle q=0$ and $\Varangle s=0$. Thus we see that $r$ lies on the shortest geodesic segment joining $p$ to $q$. But this contradicts to $r \in C(p)$.

Q.E. D.

Proof of Theorem B. The proof would be complete if we could show 
that $M$ is isometric to $P R^{n}(\delta)$ if $L(M)=3 \pi / 2 \sqrt{\delta}$. By the definitions of $L(M)$ and $d(M)$ we have $3 d(M) \geqq L(M)$. Hence we have $d(M) \geqq \pi / 2 \sqrt{\delta}$. Therefore by theorem 3 we have $d(M)=\pi / 2 \sqrt{\delta}$ and see that $M$ is isometric to $P R^{n}(\delta)$. Q.E. D.

\section{Department of Mathematics, Hokkaido University}

\section{References}

[1] H. NAKAGAWA: Riemannian manifolds with many geodesic loops, J. Math. Soc. Japan, 20 (1968) 648-654.

[2] K. Shionama: The diameter of $\delta$-pinched manifolds, J. Differential Geometry, 5 (1971) 61-74.

[3] Y. Tsukamoto: On some global properties of a Riemannian manifold with positive curvature, Sugaku, 15 (1963) 97-101, (In Japanese).

[4] Y. Tsukamoto: On the topological structure of a certain Riemannian manifold with positive curvature, Math. Rep. General Ed. Department Kyushu Univ., 3 (1965) 27-32, (In Japanese).

[5] Y. Tsukamoto: On certain Riemannian manifolds of positive curvature, Tôhoku Math. J., 18 (1966) 44-49.

(Received August 22, 1972) 